
\title{
$\begin{array}{ll}\text { Research Square } & \begin{array}{l}\text { Preprints are preliminary reports that have not undergone peer review. } \\ \text { They should not be considered conclusive, used to inform clinical practice, } \\ \text { or referenced by the media as validated information. }\end{array}\end{array}$
}

\section{A capsid with a twist: Assembly mechanism of the pleomorphic immature poxvirus scaffold}

\section{Matthias Wolf ( $\nabla$ matthias.wolf@oist.jp )}

Okinawa Institute of Science and Technology Graduate University https://orcid.org/0000-0001-84800918

\section{Jae-Kyung Hyun}

Okinawa Institute of Science and Technology https://orcid.org/0000-0003-2914-537X

Hideyuki Matsunami

Okinawa Institute of Science and Technology Graduate University https://orcid.org/0000-0001-58143766

\section{Tae Gyun Kim}

Okinawa Institute of Science and Technology Graduate University

Article

Keywords:

Posted Date: October 22nd, 2021

DOl: https://doi.org/10.21203/rs.3.rs-902523/v1

License: (c) (i) This work is licensed under a Creative Commons Attribution 4.0 International License. Read Full License

Version of Record: A version of this preprint was published at Nature Communications on March 31st, 2022. See the published version at https://doi.org/10.1038/s41467-022-29305-5. 


\section{Abstract}

In Vaccinia virus (VACV), the prototype poxvirus, scaffold protein D13 forms a honeycomb-like lattice on the viral membrane that results in formation of the pleomorphic immature virion (IV). The structure of D13 is similar to those of major capsid proteins that readily form icosahedral capsids in nucleocytoplasmic large DNA viruses (NCLDVs). However, the detailed assembly mechanism of the nonicosahedral poxvirus scaffold has never been understood. Here we show the cryo-EM structures of D13 trimer and scaffold intermediates produced in vitro. The structures reveal that the short N-terminal a-helix is critical for initiation of D13 self-assembly. The continuous curvature of the IV is mediated by electrostatic interactions that induce torsion between trimers. The assembly mechanism explains the semi-ordered capsid-like arrangement of D13 that is distinct from icosahedral NCLDVs. Our structures explain how a single protein can self-assemble into different capsid morphologies, and represents a local exception to the universal Caspar-Klug theory of quasi-equivalence.

\section{Introduction}

Poxviruses are large, enveloped, double-stranded DNA viruses that infect humans, other vertebrates, and arthropods. Viral infection typically causes skin lesions, but can also prove fatal, as in the case of smallpox. Despite eradication of smallpox, zoonotic outbreaks of smallpox-like diseases urge thorough understanding of the poxvirus replication cycle to facilitate development of strategies for prevention and treatment ${ }^{1}$.

Poxviruses share a common phylogeny and similar replication pathways with NCLDVs ${ }^{2}$. A common feature of NCLDVs is the presence of major capsid proteins with a double-jelly-roll motif that assemble into icosahedral capsids. Notable exceptions are poxviruses, ascoviruses, and pandoraviruses, which exhibit variable capsid diameters and do not follow canonical symmetry ${ }^{3,4}$. In Vaccinia virus (VACV), copies of the double-jelly-roll protein D13 bind to the viral membrane via interaction with its partner protein, A17, and assemble into a scaffold surrounding the viral membrane $e^{5,6}$. This process leads to formation of the spherical immature virion (IV). Subsequent proteolytic processing of A17 releases the D13 scaffold from the viral membrane, leading to a dramatic transformation into the brick-shaped mature virion (MV). While a set of proteins have been identified as essential for formation of poxvirus IV, selfassembly of D13 has been proposed as the sole determinant that governs shape and size of IVs ${ }^{7-9}$.

The $62-\mathrm{kDa}$ D13 protein exists as trimers in vivo and in vitro, and structures of the recombinantly expressed D13 trimer have been determined by X-ray crystallography ${ }^{9-12}$. D13 adopts a double-jelly-roll structure, composed of 8 antiparallel $\beta$-strands. A head domain is inserted between $\beta$-strands of the Cterminal jelly roll. Recently, the structure of D13 in complex with the antibiotic, rifampicin, was determined by X-ray crystallography, illustrating the mechanism by which the anti-viral drug prevents binding of $A 17$, occupying the phenylalanine-rich pocket on the 3-fold axis of the D13 trimer ${ }^{11}$. Inhibition of the D13-A17 interaction would impair recruitment of D13 onto the viral membrane, leading to aberrant virion 
formation, as previously demonstrated by mutagenesis ${ }^{13-17}$. However, none of the crystallographic packing arrangements from any of the reported structures represent the honeycomb-shaped lattice observed on the surface of authentic IV, and inter-trimer interactions that govern D13 assembly have been poorly resolved. Low-resolution electron microscopy and three-dimensional (3D) reconstructions obtained from negatively stained two-dimensional (2D) planar arrays of D13 and its orthologue, orfv075 protein, from orf virus, provided the overall organization of trimers in the context of the honeycomb-shaped lattice ${ }^{9,18}$. Nevertheless, molecular details of the inter-trimer interface were inconclusive in these studies. Here we show the cryo-EM structures of D13 trimers, trimer doublets, and the tubular assembly of an expanded honeycomb lattice produced in vitro. The structures reveal that the short $\mathrm{N}$-terminal a-helix is critical for the initiation of D13 self-assembly. The continuous curvature of the immature virion is mediated by electrostatic interactions that induce torsion between the trimers. The results explain the mechanism of semi-ordered capsid-like arrangement of D13 that is distinct from homologous capsid proteins of icosahedral NCLDVs. Our structures demonstrate how a single protein can self-assemble into different capsid morphologies, induced by a small conformational change of a peripheral helix.

\section{Results}

\section{Structure of D13 trimer}

We purified recombinant D13 trimer protein for structure determination using single-particle cryo-electron microscopy (cryo-EM). Initial attempts were hampered by problems associated with preferred particle orientation and spontaneous protein assembly into honeycomb-like crystalline patches that consist of concentric rings of five or six trimers (Supplementary Fig. 1). This problem was mitigated using holey EM grids coated with a graphene oxide support film (Supplementary Fig. 2a) and the cryo-EM structure of D13 trimer was determined at $2.3 \AA$ resolution (Fig. 1a and Supplementary Fig. 2), which exceeds the resolution of even the best available crystal structures ${ }^{9-11}$. In our atomic model, we followed the convention for the jelly-roll fold with BIDG-CHEF $\beta$-strand-order (Fig. 1b). The refined atomic model based on this structure was largely identical to previously reported crystal structures (average RMSD 0.495 $\AA)^{9-11}$. However, the presence of the short N-terminal tail a-helix (aa1-10) was inconsistent with previously reported models. In the first two crystal structures, electron density for this helix was only observed in the D513G mutant, but not in wild type protein ${ }^{9,10}$, whereas subsequently reported crystal structures contain the corresponding density in only two of the three subunits ${ }^{11}$. Our cryo-EM map showed intact $\mathrm{N}$-terminal tail a-helices in all three subunits, with or without imposition of 3-fold symmetry, although reduced local map resolution and the increased B-factor suggested that the helix was prone to dislocation (Supplementary Fig. 2f-h). In our structure, the hydrophobic face of the N-terminal helix (M1, 15, 16 and L9) was held in a pocket of surrounding hydrophobic residues from the adjacent subunits. Additional hydrogen bonds were identified between the side chains of T4, N2, Q30 and carboxylic groups of L452, V451, N2 (Fig. 1c).

\section{Inter-trimer interactions in D13 trimer doublet}


Image processing of D13 trimers revealed a small sub-population of adjacent trimers bound together, forming doublets of trimers. Further 2D and 3D classifications enabled segregation of doublet particle images from the trimer singlets. The scarcity of intermediate angular views within the dataset was supplemented by additional micrographs acquired from tilted specimen grids without the graphene oxide support film (Supplementary Fig. 3a). Although resolution of the resulting 3D reconstruction (3.9 ̊ on average) was not fully isotropic (Supplementary Data Fig. 3e), it allowed for unambiguous positioning of an atomic model for identification of the inter-trimer interfaces. There are two major interfaces that mediate doublet formation (Fig. 2a). The first is located between one of the head domains on each of the two trimers. Although map resolution at this interface was insufficient to clearly define side chain interactions, it was possible to trace the loops extending toward the neighboring trimer (Fig. 2b). These include an extended loop that connects $\beta$-strands $E$ and $F\left(E_{H}\right.$ loop) and a hairpin loop between $\beta$ strands $C$ and $D\left(C D_{H}\right.$ loop) in the head domain. The interface is clustered with polar $(N 355, Q 324, S 356)$ and charged amino acid residues (D325, R353, K354) around the two-fold symmetry axis. Side chains of R353 in the $\mathrm{EF}_{\mathrm{H}}$ loop and D325 in the $\mathrm{CD}_{\mathrm{H}}$ loop extend toward each other, suggesting salt bridge interactions. Another interface is located at the base of the trimers, which would be proximal to the viral membrane in the IV. At this interface, the major difference of doublet structure in comparison to the singlet trimer is the unambiguous absence of the $\mathrm{N}$-terminal tail a-helix (Fig. 2c). The interface involves the loop that connects $\beta$-strands $C$ and $D$ of the $N$-terminal jelly-roll $\left(C D_{N}\right.$ loop) and the loop connecting $\beta$ strands $\mathrm{G}$ and $\mathrm{H}$ of the $\mathrm{C}$-terminal jelly-roll $\left(\mathrm{GH}_{\mathrm{C}}\right.$ loop) in the neighboring trimer. Unlike the head-to-head interface, this interaction occurs pairwise between two monomers in each of the two trimers. Clearly resolved features in the reconstructed electron potential map between Y62 and R498 suggest cation- $\pi$ interactions as the main stabilizer at this interface. We found $\mathrm{Y} 62$ in close proximity to 16 of the Nterminal tail a-helices in the singlet trimer structure, suggesting that the weak hydrophobic interaction between these residues may have been altered in favor of inter-trimeric interaction in the doublet structure, upon dislocation of the $\mathrm{N}$-terminal tail a-helix. Furthermore, the doublet is characterized by a slight twist of the trimer axes defined by a torsion angle of $8.0^{\circ}$, leading to an arched arrangement when the doublets are linearly expanded (Fig. 2d, e). The bases of D13 trimers face the center of the arch and this supports the idea that remodeling of the viral membrane into a convex curvature is induced by the self-assembled D13 scaffold. However, planar expansion of the doublet prohibits closure of the domeshaped curvature, suggesting that additional inter-trimer interfaces or oligomers are involved in formation of spherical poxvirus scaffolds (Fig. 2f).

\section{Critical role of $\mathrm{N}$-terminal a-helix in poxvirus scaffold assembly}

The absence of the N-terminal tail a-helix in the doublet structure suggests that its displacement likely helps to initiate inter-trimer interactions, promoting ordered assembly of oligomers and leading to formation of spherical IV-like particles. To test this hypothesis, we used in vitro D13 self-assembly. The protein solution turned turbid after dialysis into low-salt buffer, but examination by transmission electron microscopy (TEM) showed aggregated honeycomb-like patches and rodlets without any IV-like particles (Fig. 3a). Under the same conditions, the protein with an $\mathrm{N}$-terminal $\mathrm{His}_{6}$-tag and an extended linker (28 
amino acid residues) assembled exclusively into spherical particles that mimic the size and shape of the authentic VACV IV scaffold, with a distinctive honeycomb-shaped pattern (Fig. 3b and Supplementary Video 1). These observations were previously described, although the rationale behind this assembly discrepancy could not be explained ${ }^{9}$. To investigate whether the $\mathrm{N}$-terminal tail a-helix is indeed responsible for this observation, we determined the structure of His ${ }_{6}$-tagged D13 protein at $2.6 \AA$ resolution by cryo-EM. As expected, the $\mathrm{N}$-terminal tail helix feature was nearly absent in the reconstructed map, possibly due to the additional charges on the N-terminal His-tag that perturb the hydrophobic environment that stabilizes this interaction in native, untagged protein. Furthermore, we expressed and purified mutant protein (D13 $18-548$ ) with a truncation of $17 \mathrm{~N}$-terminal amino acid residues, including the $\mathrm{N}$-terminal tail a-helix. Our cryo-EM reconstruction of the mutant displayed no significant change from the wild-type D13 (wtD13) or D13 with an intact $\mathrm{N}$-terminal His 6 -tag. This mutant assembled into tubular and spherical structures that exhibit a honeycomb-like lattice (Fig. 3c) under lowsalt conditions in a fashion similar to the $\mathrm{His}_{6}$-tagged protein, thereby confirming the essential function of the $\mathrm{N}$-terminal tail helix for D13 self-assembly.

\section{Inter-trimer interactions in tubular D13 assembly}

In order to investigate inter-trimeric interactions in the context of higher-order assembly, tubular assembly products of the $\mathrm{N}$-terminal truncation mutant D13 $18-548$ were subjected to cryo-EM and helical reconstruction (Supplementary Fig. 4a-d). Refined helical parameters indicated a 3-start helix with a 33.9Å rise and a $77.0^{\circ}$ twist. A 3D reconstruction at 7.3 Å resolution exhibited a continuous honeycombshaped lattice rolled into a tube (Fig. 4a). Single-particle analysis of symmetry-expanded, signalsubtracted particle images of trimer sextets resulted in improved cryo-EM map resolution at $3.9 \AA$, enabling further analysis of inter-trimer interfaces (Fig. 4b and Supplementary Fig. 4e-h). The structure exhibits two alternating modes of inter-trimer arrangements, which are mostly stabilized by electrostatic interactions (Supplementary Fig. 5) and agrees with the self-assembly behavior of D13 with varying salt concentration of the buffer solution. The first mode (mode I) is similar to the doublet structure in which the interaction between the head domains appears most extensive between the $\mathrm{EF}_{\mathrm{H}}$ and $\mathrm{CD}_{\mathrm{H}}$ loops. The base-to-base interface involves cation- $\pi$ interaction between $\mathrm{Y} 62$ of the $\mathrm{CD}_{\mathrm{N}}$ loop and $\mathrm{R} 498$ of $\mathrm{GH}_{\mathrm{C}}$ loop (Fig. 4c). The second mode (mode II) also implicates two inter-trimer interfaces. However, in this arrangement, two monomers in each trimer are involved, forming pairwise interactions (Fig. 4d). In one moiety, contacts between the $\mathrm{EF}_{\mathrm{H}}$ and $\mathrm{FG}_{\mathrm{N}}$ loops (P144 and N145) appeared most prominent. In the other moiety, the $\mathrm{EF}_{\mathrm{H}}$ loop is in close proximity to charged residues in a short $\beta$-strand in the $\mathrm{DE}_{\mathrm{N}}$ loop (E77 and K79) and a short a-helix in the $\mathrm{HI}_{\mathrm{N}}$ loop (E205 and K204). Symmetric Y62-R498 interactions were no longer found in the base-to-base interface of the mode II arrangement. One moiety appears to involve a cation- $\pi$ interaction between $\mathrm{Y} 62$ and R446 in $\beta$-strand $E$ of the C-terminal jelly roll $\left(E_{c}\right)$, replacing R498 in the mode I arrangement. The other moiety exhibits close contact between R498 of one monomer with Y 62 and D60 of the other monomer. Residues R353 and Y62 were critical in all modes of arrangements for head-to-head and base-to-base interfaces, respectively. This was confirmed by mutation of these residues to alanine, resulting in assembly incompetence in low-salt buffer (Supplementary Fig. 6). Each 
trimer was positioned with a slight torsion in relation to neighboring trimers in the sextet. Torsion angles ranged from $7.6^{\circ}$ to $9.4^{\circ}$, and from $12.5^{\circ}$ to $13.5^{\circ}$ in mode I and mode II arrangements, respectively (Fig. 4 e). The torsion angle varied among mode I arrangements, indicating slight plasticity. While twist direction was consistent for mode I arrangements, it alternated depending on the asymmetric contact points between the trimers. Variability of the inter-trimer torsion angle and direction found in the helical assembly suggests a possible continuous curvature in all directions, inducing the spherical morphology observed in authentic VACV IV. To examine this, two types of inter-trimer arrangements were alternatively modeled, instead of two consecutive mode I arrangements, followed by a mode II, as originally found in the tubular assembly. This allowed for closure of a crown-shaped concentric ring of six trimers (Fig. 4f). However, expansion of the sextet failed to generate a continuous spherical morphology.

\section{Discussion}

Poxvirus scaffold consists of loosely connected concentric rings of six trimers in contrast to tightly packed capsomers of icosahedral NCLDV capsids that require additional minor capsid proteins and accessory proteins ${ }^{19-22}$. Poxviruses lack such accessory proteins but the construction of the scaffold is solely based on D13 self-assembly as shown in this study. The charge distribution on the surface of D13 trimer roughly resembles those of capsomers of NCLDVs, hence likely to follow their capsid assembly scheme based on the electrostatic interactions ${ }^{23}$. The inter-trimeric torsion and its variation are the unique features of the poxvirus scaffolding protein. We revealed that the curvature that results from alternating torsion between the D13 trimers forms the basis of semi-regular, spherical immature poxvirus particles. Mild curvature between the capsomers has been described for African swine fever virus (ASFV) major capsid protein although such curvature depends on the microenvironment within the icosahedron, and dissimilar to continuous curvature in the case of poxvirus scaffold ${ }^{20}$

We found that the N-terminal short tail a-helix of D13 is critical for in vitro assembly into ordered structures. We propose that repositioning of the $\mathrm{N}$-terminal tail a-helix away from D13 toward the surface of the lipid bilayer may act as a molecular trigger that initiates scaffold assembly in vivo. This scenario implies that D13 assembly into a scaffold occurs in situ only when bound to the viral membrane via D13A17 interaction, but not before, thereby preventing the virion assembly sequence without a lipid membrane. This is supported by cellular cryo-electron tomography (cryo-ET) of immature poxvirus, which showed that intact scaffold formation requires association with a lipid membrane ${ }^{8}$. N-terminal helices have been found to act as anchors on proximal lipid membranes in double-jelly-roll capsid proteins of dsDNA bacteriophages and African swine fever virus (ASFV) ${ }^{24-26}$. However, such interactions were only proposed to stabilize the capsid, but not to initiate assembly.

The inter-trimer interfaces are distal from the rifampicin binding site and from residues that confer rifampicin resistance in VACV, indicating that these inter-trimeric interactions are independent from drug binding ${ }^{11,14}$ (Supplementary Fig. 7a). Aspartic acid residues 513 (D513) from neighboring trimers were 
within $7 \AA ̊$ along the 2-fold symmetry axis in the mode I arrangement, but mutation D513G, which leads to abrogation of the curved lattice ${ }^{12}$, could not be explained by our structure (Supplementary Fig. $7 b$ ).

Nearly 60 years ago, Donald Caspar and Aaron Klug introduced their theory of quasi-equivalence of macromolecular assemblies, which predicts arrangements of protein subunits at the surface of icosahedral virus particles in a regular lattice, parametrized by a triangulation number $(T)$. The CasparKlug theory has been instrumental in understanding molecular symmetry of icosahedral viruses and many other macromolecular assemblies. With the advent of cryo-EM at near-atomic resolution, a number of deviations from ideal symmetry have been discovered ${ }^{27-29}$. The VACV scaffold is an example of such a deviation, in which D13 is uniquely responsible for generating various building blocks and continuous curvature. The observation of pentameric tiling (Fig. 5a and Supplementary Fig. 1) supports the hypothesis that the underlying symmetry of the VACV scaffold as a result of D13 assembly is an icosahedral lattice, but local deviations form ideal Caspar-Klug tiling result in an imperfect, yet semiregular scaffold (Fig. 3b and Supplementary Video 1). The N-terminal helix represents a mobile element that regulates initiation of scaffold assembly and electrostatic inter-trimer interactions, resulting in different local symmetries that follow the principle of quasi-equivalence within a patch of common assembly mode, but not throughout the entire scaffold (Fig. 5b-d). Using only a single gene product, such locally irregular transient scaffold assembly may serve as an economic and efficient way of packaging the large poxvirus genome in its immature virion. The novel interactions identified in our study may inspire the design of a new generation of assembly inhibitor drugs (e.g., peptidomimetics based on the Nterminal helix) and further structural investigation of other pleomorphic viral capsids, such as HIV.

\section{Declarations}

\section{Acknowledgements}

We thank Dr. Fasséli Coulibaly for critical reading of our manuscript. We are grateful to Dr. Steven Aird for technical editing. We appreciate support by the Scientific Computing and Data Analysis Section (OIST RSD). This work was supported by the Platform Project for Supporting Drug Discovery and Life Science Research (BINDS) from AMED under grant number JP18am0101076 (to M.W.), and by a JSPS KAKENHI Grant JP17K07318 (to HM) and 21K06039 (to JH). MW was supported by direct funding from Okinawa Institute of Science and Technology Graduate University.

\section{Author contributions}

$\mathrm{JH}$ conceived and designed experiments. JH and TK performed molecular cloning and protein purification. JH created mutants. JH and MW collected cryo-EM data. JH processed cryo-EM data. HM and $\mathrm{JH}$ carried out atomic modeling and refinement into cryo-EM maps. MW supervised the project and provided advice on cryo-EM and image processing. JH and MW wrote the manuscript. All authors analyzed results and contributed to writing the paper.

\section{Declaration of Interests}


The authors declare no competing interests.

\section{Data availability}

Atomic coordinates of cryo-EM based models have been deposited under PDB IDs 7VFD, 7VFE, 7VFF, 7VFG and 7VFH. Cryo-EM maps have been deposited in the EM database under accession code EMD31949, EMD-31950, EMD-31951, EMD-31952, EMD-31953 and EMD-31954.

\section{Methods}

\section{Protein expression and purification}

VACV D13 was prepared as previously described by Hyun et al. ${ }^{9}$. Briefly, the D13 gene in the pPROEX-Hta vector was expressed in BL21(DE3) cells and purified by immobilized metal affinity chromatography (IMAC) using HisTrapHP (Cytiva). The $\mathrm{N}$-terminal $\mathrm{His}_{6}$-tag was removed by TEV protease digestion and products were further purified by IMAC to remove remaining tagged protein. TEV protease digestion was omitted for production of $\mathrm{His}_{6}$-tagged protein used in D13 self-assembly experiments. The protein was further purified by size exclusion chromatography in a buffer containing $50 \mathrm{mM}$ Tris- $\mathrm{HCl}(\mathrm{pH} 8.0), 600$ $\mathrm{mM} \mathrm{NaCl}, 50 \mathrm{mM}$ L-arginine, $50 \mathrm{mM} \mathrm{L-glutamic} \mathrm{acid} \mathrm{and} 2 \mathrm{mM} \beta$-mercaptoethanol.

\section{Mutagenesis}

The N-terminal truncation mutant (D13 $18-548$ ) was generated by PCR using a forward primer lacking the leading 51 bases of the D13 DNA sequence. Point mutations R353A and Y62A were introduced to wtD13 and D13 ${ }_{18-548}$ using a Q5 site-directed mutagenesis kit (New England Biolabs Inc.). Expression and purification procedures for the mutant were identical for those of wtD13. Primers used for mutagenesis are listed in Supplementary Information.

\section{D13 self-assembly}

A $100-\mu \mathrm{L}$ aliquot of purified protein at approximately $2 \mathrm{mg} / \mathrm{mL}$ was dialyzed in buffer containing $10 \mathrm{mM}$ Tris- $\mathrm{HCl}\left(\mathrm{pH} \mathrm{8.0),} 150 \mathrm{mM} \mathrm{NaCl}\right.$, and $2 \mathrm{mM} \beta$-mercaptoethanol, at $4^{\circ} \mathrm{C}$ for at least $12 \mathrm{~h}$. The resulting turbid solution was centrifuged at $15,000 \mathrm{rpm}$. Then $80 \mu \mathrm{L}$ supernatant were removed and the pellet was resuspended using the remaining solution. The pellet sample was negatively stained with $2 \%$ uranyl acetate and examined using TEM to confirm the assembly. The remaining supernatant was checked by TEM to confirm presence of homogeneous trimers. This preparation was used for cryo-EM of singlet trimer particles.

\section{Negative staining and transmission electron microscopy}

Routine TEM examinations were performed using negatively stained samples. $5 \mu \mathrm{L}$ of protein at approximately $0.01 \mathrm{mg} / \mathrm{mL}$ were loaded onto freshly glow-discharged EM grids with continuous carbon support films. $90 \mathrm{sec}$ were allowed for sample adsorption and then grids were washed with three droplets 
of distilled water. $5 \mu \mathrm{L}$ of $2 \%$ uranyl acetate solution were loaded onto grids, followed by 60 -sec incubation. A piece of filter paper was used to blot excess stain solution and grids were air-dried. Specimen grids were examined using a Talos L120C TEM equipped with Ceta CMOS detector (Thermo Fisher Scientific (TFS)), operating at $120 \mathrm{kV}$ acceleration voltage.

\section{Cryo-electron microscopy}

For wtD13 trimers, His $_{6}$-tagged D13 trimers and D13 $18-548$ trimers, $3 \mu \mathrm{L}$ of protein at $0.12 \mathrm{mg} / \mathrm{ml}$ were loaded onto holey EM grids (Quantifoil R1.2/1.3 Cu300, Quantifoil Micro Tools GmbH) prior treated with graphene oxide film flakes (Sigma Aldrich). The sample was vitrified on a Vitrobot Mark IV (TFS) operating at $4^{\circ} \mathrm{C}$ and $>90 \%$ relative humidity. wtD13 image data were acquired with a Titan Krios TEM (TFS) operating at $300 \mathrm{kV}$ at a nominal magnification of $155,000 \mathrm{x}$, corresponding to $0.518 \AA \mathrm{A} / \mathrm{pixel}$ at the specimen level, with the defocus ranging between $0.5 \mu \mathrm{m}$ and $1.5 \mu \mathrm{m}$. Spot size $8, \mathrm{C} 2$ aperture $70 \mu \mathrm{m}, \mathrm{OL}$ aperture $100 \mu \mathrm{m}$ and $1.8 \mu \mathrm{m}$ illumination area were used. 50 movie fractions were collected on a Falcon 3EC direct electron detector in electron counting mode using EPU software (TFS), with a total electron dose of $50 \mathrm{e}^{-} / \AA^{2}$, and dose rate of $1.6 \mathrm{e}^{-} / \AA^{2} / \mathrm{sec}$. $\mathrm{His}_{6}$-tagged D13 image data were collected with a Titan Krios TEM (TFS) operating at $300 \mathrm{kV}$ at a nominal magnification of $155,000 \mathrm{x}$, corresponding to 0.518 $\AA /$ pixel at the specimen level, with the defocus ranging between $0.5 \mu \mathrm{m}$ and $1.5 \mu \mathrm{m}$. Spot size $8, \mathrm{C} 2$ aperture $70 \mu \mathrm{m}, \mathrm{OL}$ aperture $100 \mu \mathrm{m}$ and $1.8 \mu \mathrm{m}$ illumination area were used. 50 movie fractions were collected on a Falcon 3EC direct electron detector in electron-counting mode using EPU software (TFS), with a total electron dose of $50 \mathrm{e}^{-} / \AA^{2}$, and dose rate of $1.6 \mathrm{e} / \AA^{2} / \mathrm{sec}$. D13 $18-548$ data were collected on a Talos Arctica TEM (TFS) operating at $200 \mathrm{kV}$ at a nominal magnification of 92,000x, which corresponds to $1.12 \AA$ /pixel at the specimen level, with defocus ranging between $0.6 \mu \mathrm{m}$ and $1.2 \mu \mathrm{m}$. The spot size was 9. A 70- $\mu \mathrm{m} \mathrm{C2}$ aperture and 100- $\mu \mathrm{m} \mathrm{OL}$ aperture were used. Exposures were acquired as movies of 50 dose fractions on a Falcon 3 direct electron detector in EC mode using EPU software (TFS), with a total electron dose of $50 \mathrm{e}^{-} / \AA^{2}$ at a dose rate of $0.6 \mathrm{e} / \AA^{2} / \mathrm{sec}$.

For D13 doublets, multiple datasets were collected in order to enrich the angular orientation of particle images. The first two datasets were collected from a grid prepared as for the D13 trimer singlet, using holey carbon grids with additional graphene oxide film. For the third and fourth datasets, samples were prepared on gold grids (UltrAuFoil R1.2/1.3 300, Quantifoil Micro Tools GmbH) without a graphene oxide support film. Vitrification methods were the same for all grid preparations. For the third and fourth datasets, in which D13 trimers were preferentially oriented and partially assembled, the microscope stage was tilted to $30^{\circ}$ and $45^{\circ}$, respectively. Image data were collected on a Talos Arctica TEM (TFS) operating at $200 \mathrm{kV}$ at a nominal magnification of $92,000 \mathrm{x}$, which corresponds to $1.12 \AA /$ pixel at the specimen level, with defocus ranging between $0.5 \mu \mathrm{m}$ and $5.0 \mu \mathrm{m}$. The spot size was 9 or 10 . A 50- or 70- $\mu \mathrm{m} \mathrm{C2}$ aperture and $100-\mu \mathrm{m} \mathrm{OL}$ aperture were used. Exposures were acquired as movies of 50 dose fractions on a Falcon 3 direct electron detector in electron-counting mode using EPU software (TFS), with a total electron dose of $50 \mathrm{e}^{-} / \AA^{2}$ at a dose rate between $0.6-0.9 \mathrm{e}^{-} / \AA^{2} / \mathrm{sec}$. 
For the tubular D13 assembly, $3 \mu \mathrm{L}$ of resuspended pellet from the assembly solution were loaded onto a holey EM grid (Quantifoil R2/2 Cu300). Vitrification methods were the same as above. Data were acquired on a Titan Krios TEM (TFS) operating at 300kV in EFTEM mode (Gatan Quantum 968) at a nominal magnification of 105,000 x corresponding to $1.4 \AA$ pixel at the specimen level, with defocus

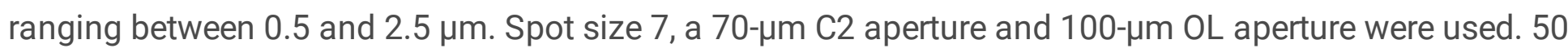
dose fractions/movie were recorded with a K2 Summit direct electron detector (Gatan) and EPU software (TFS), with a total electron dose of $50 \mathrm{e}^{-} / \AA^{2}$ at a dose rate of $5 \mathrm{e}^{-} / \AA^{2} / \mathrm{sec}$.

For cryo-ET of spherical IV-like particles, assembly solutions that resulted from introducing $\mathrm{His}_{6}$-tagged D13 into low-salt buffer were mixed with 10-nm gold fiducial marker (AURION) in a 4:1 (v:v) ratio. $3 \mu \mathrm{L}$ of the mixture were loaded onto a non-glow-discharged holey EM grid (Quantifoil R2/2 Cu300) and vitrified on a Vitrobot Mark IV (TFS) operating at $4^{\circ} \mathrm{C}$ and $>90 \%$ relative humidity. Tomographic data were acquired on a Titan Krios TEM (TFS) operating at $300 \mathrm{kV}$ at a nominal magnification of $45,000 \mathrm{x}$ corresponding to $2.26 \AA$ Apixel at the specimen level, defocused between 5.0 and $6.0 \mu \mathrm{m}$. The spot size was 9, and a 70- $\mu \mathrm{m} \mathrm{CL}$ aperture, with a $100-\mu \mathrm{m}$ OL aperture and a 1.8- $\mu \mathrm{m}$ beam diameter area were used. A dose-symmetric tomography acquisition scheme was applied on a Falcon 3 direct electron detector in EC mode using Tomo 4 software (TFS) over $+/-63^{\circ}$ tilt with $3^{\circ}$ intervals. The total dose applied to each tomogram was approximately $120 \mathrm{e}^{-} / \AA^{2}$, and 5 movie fractions were collected for each tilt image at a dose rate $0.14 \mathrm{e}^{-/} \AA^{2} / \mathrm{sec}$.

\section{Cryo-EM Image processing}

All single-particle datasets were processed with Relion 3.1 software, unless stated otherwise ${ }^{30-33}$. Motion correction and contrast transfer function (CTF) estimation were performed using MotionCor2 ${ }^{34}$ and CTFFIND $4^{35}$, respectively. Falcon 4 movie data in EER format from the Krios G4 were processed with Relion 3.1.2, Statistics of individual datasets can be found in Supplementary Table 1. Cryo-ET data were processed using IMOD. Visual examination of maps and figure preparations were done with UCSF Chimera $^{36}$, Chimera $X^{37}$ and PyMol (The PyMol Molecular Graphic System, Version 2.4.0, Schrödinger, LLC).

For wtD13 trimers, 2,168 dose-weighed micrographs were manually inspected. Images without graphene oxide support or poor estimated maximum resolution or strong astigmatism from CTFFIND calculation were discarded. From 1,862 assorted micrographs, particles were automatically picked using 2D class averages of manually picked particles as a template. 668,437 particles were down-sampled by 4 and extracted into 128 pixel squared boxes. The particles were then subjected to two rounds of 2D classification to eliminate poorly aligned particle images (Supplementary Fig. 2a). The resulting 204,563 particles were re-extracted from micrographs with a down-sampling factor of 2, into 256 pixel squares boxes. A consensus reconstruction was generated by refinement without imposition of symmetry. Then, 3D classification was performed without particle alignment, from which 130,384 particles that belong in the class exhibiting the best structural details were selected for the final round of refinement (Supplementary Fig. 2b). Further refinements on the assorted particles were performed with C3 symmetry 
imposition. CTF refinement for magnification anisotropy, optical aberrations and per-particle defocus were performed, followed by Bayesian particle polishing. Final 3D refinement and map sharpening resulted in a reconstruction at $2.25 \AA$ spatial resolution based on 0.143 Fourier shell correlation (FSC) criterion of independently refined halfset reconstructions (Supplementary Fig. 2c-e). The particles that had been selected from the 3D classification were also subjected to 3D refinement without symmetry imposition, CTF refinement and Bayesian polishing. The final resolution of the unsymmetrized 3D reconstruction was estimated at $2.63 \AA$ resolution ( $\mathrm{FSC}=0.143$ ).

For $\mathrm{His}_{6}$-tagged D13 trimers, 1,328 dose-weighed micrographs were selected from 1,418 total micrographs after removing micrographs with poor estimated maximum resolution or strong astigmatism. 284,340 particles were semi-automatically picked, down-sampled by 4 , and boxed in $128-$ pixel squared boxes. Two rounds of 2D classifications were performed to remove poorly aligned images. The resulting 216,590 particles were re-extracted from micrographs with a down-sampling factor of 2 into 256-pixel squared boxes, resulting in a sampling interval of $1.036 \AA$ /pixel. 3D classification was performed without symmetry imposition and only particles that belong in classes with detailed structural features were selected. The resulting 173,354 particles were subjected to 3 rounds of 3D refinement with C3 symmetry. CTF refinement and particle polishing were performed between refinements. The final resolution of the $3 \mathrm{D}$ reconstruction was estimated at $2.63 \AA$ (FSC=0.143).

For D13 18-548 trimers, 1,112 dose-weighed micrographs out of 1,227 total micrographs were selected after removing micrographs with poor estimated maximum resolution or strong astigmatism. 743,834 particles were semi-automatically picked, binned by 2 , and boxed in 128-pixel squared boxes. A 2D classification was performed to remove poorly aligned images, and 475,652 good particles were re-extracted from unbinned micrographs (1.12 $\AA$ /pixel), into 256 -pixel squares boxes. 3D classification was performed without symmetry imposition and 156,813 particles that belong in classes with detailed structural features were selected. 3 rounds of 3D refinement with C3 symmetry were performed, with CTF refinement and particle polishing between. The final resolution of the 3D reconstruction was estimated at $4.10 \AA$ Å resolution ( $\mathrm{FSC}=0.143)$.

For D13 trimer doublets, four datasets were used (550, 280, 676 and 189 movies each), in which each movie dataset was independently processed for beam-induced motion correction, CTF estimation, and particle auto-picking. Particle auto-picking was performed using a template 3D map produced by artificially joining two copies of D13 trimer maps. To minimize the effect of reference bias on the highresolution signal imposed by particle picking, the template was low-pass filtered to $20 \AA$. 835,797 particles from combined 1,695 micrographs were down-sampled by a factor of 2 and extracted into 128pixel squared boxes. Particle images were subjected to 2D class averaging, from which images that belong in classes with poor structural features were eliminated (Supplementary Fig. 3a). The resulting 746,600 particles were subjected to 3D classification without symmetry imposition, using an initial 3D reference generated from image data. The majority of particles partitioned into 3D classes with a pronounced trimer singlet featuring an adjacent ghost-like density of an extra trimer (Supplementary Fig. 3b). Only 164,259 particles from a 3D class that clearly exhibited the trimer doublet were selected for 
further processing. Particle images were re-extracted from micrographs into 256-pixel squared boxes without down-sampling. Because of clear 2-fold symmetry of the 3D class, C2 symmetry was imposed in subsequent steps. The initial 3D refinement resulted in a 3D reconstruction at $4.78 \AA$ at $0.143 \mathrm{FSC}$. Then, CTF refinement for magnification anisotropy, optical aberrations, and per-particle defocus was performed on each optical group, followed by Bayesian particle polishing. These procedures were repeated three times. Next, the aligned particles were 3D-classified without further image alignment, from which 42,854 particles that belong in classes with detailed structural features were selected (Supplementary Fig. 3b). These particles were re-extracted into 512-pixel squared boxes to include the delocalized signal from highly defocused images (up to $5 \mathrm{~mm}$ underfocus). 3D refinement, CTF refinement, and particle polishing were repeated twice. The resolution of the final 3D reconstruction was $3.87 \AA$ at 0.143 FSC (Supplementary Fig. 3d). 3D resolution anisotropy of the reconstruction was calculated using the 3DFSC server (https://3dfsc.salk.edu/) ${ }^{38}$. The resulting estimate indicates global resolution at $4.55 \AA$ at 0.143 FSC and sphericity of 0.769 (Supplementary Fig. 3e).

For the D13 tubular assembly, 7,621 movies were motion-corrected followed by CTF estimation. Micrographs with poor estimated maximum resolution and severe astigmatism were discarded, based on the CTFFIND4 calculation. From 7,529 micrographs, start and end coordinates of the tubes were manually selected, and helical segments with a $167.5-\AA \AA$ inter-box distance ( $5^{\prime}$ the estimated helical rise of $33.5 \AA$ ) were down-sampled by 4 and extracted into 256 -pixel squared boxes. 194,960 particle images were subjected to 2D classification, from which 129,652 images belonging to class averages with good structural details were selected. Helical parameters were estimated by manual analysis of the helical layer-line pattern from the 2D class averages (Supplementary Fig. 4d). The images were classified in 3D, using an artificial canonical helix reference consisting of spheres generated from helical parameters $\left(77^{\circ}\right.$ twist, $33.5 \AA$ Aise with the command relion_helix_toolbox --simulate helix -o ref.mrc -subunit_diameter 140 -cyl_outer_diameter 900 -angpix 5.6 -rise 33.5 -twist 77 -boxdim 256). 75,070 particle images belonging to the $3 \mathrm{D}$ class with the best structural features were re-extracted into 512-pixel squared boxes after down-sampling by a factor of 2 (Supplementary Fig. 4b). After 3D refinement, CTF refinement and Bayesian particle polishing were performed, followed by reconstruction with Ewald sphere correction ${ }^{31,39}$. The final map resolution was $7.3 \AA$ at 0.143 FSC (Supplementary Fig. 4c). Particles were re-extracted into 1024-pixel squared boxes and a 3D reconstruction was generated prior to symmetry expansion and signal subtraction. From the helical reconstruction, 5 asymmetric units were symmetry-expanded based on the final estimated helical operator $\left(76.98^{\circ}\right.$ twist, $33.86 \AA$ rise). For signal subtraction, a volume segment that corresponds to a concentric ring of six trimers in the tube were generated from the reconstruction using UCSF Chimera. The map was then used to create a binary mask embracing six trimers, which was applied to the symmetry-expanded stack of particle images. Signal-subtracted sextet images were subjected to $2 \mathrm{D}$ class averaging using cisTEM ${ }^{40}$, and 303,052 particles from good class averages were selected for auto-refinement in 3 classes (Supplementary Fig. 4e-f). 247,311 particles from 3D classes with detailed structural features were selected for final refinement in cisTEM. The resulting half-maps were examined and post-processed using Relion 3.1. Final resolution of the map was $3.9 \AA$ at 0.143 FSC (Supplementary Fig. 4g-h). 
For Cryo-ET data, movie frames were aligned and summed using MotionCor2. Images were phase-flipped using ctfphaseflip ${ }^{41}$ and the tilt series was aligned based on gold fiducial markers. Tomograms were reconstructed from images that were binned by 2 , using simultaneous iterative reconstruction technique (SIRT) in IMOD 42 .

\section{Protein structure modeling}

$3 \mathrm{D}$ reconstructions were subjected to density modification with the ResolveCryoEM tool ${ }^{43}$ in the Phenix software suite prior to model refinement. All coordinate refinements were performed using the real-space refinement routine in Phenix ${ }^{44}$. The X-ray crystal structure of D13 (PDB ID 6BEI) was manually fitted into the cryo-EM map of our D13 trimer in UCSF Chimera, and used as a reference model for atomic coordinate refinement in Phenix, while enforcing a non-crystallographic symmetry constraint. The resulting refined trimer model was then used as a reference to refine models of the trimer doublet and sextet. These refined models were manually inspected and adjusted in $\operatorname{Coot}^{45}$, followed by final refinement with Phenix. The quality of the final models and map-to-model correlations were calculated using Phenix' Cryo-EM validation and Mtriage tools ${ }^{46}$.

\section{References}

1. Meyer, H., Ehmann, R. \& Smith, G. L. Smallpox in the Post-Eradication Era. Viruses 12, doi:10.3390/v12020138 (2020).

2. Koonin, E. V. \& Yutin, N. Evolution of the Large Nucleocytoplasmic DNA Viruses of Eukaryotes and Convergent Origins of Viral Gigantism. Adv Virus Res 103, 167-202, doi:10.1016/bs.aivir.2018.09.002 (2019).

3. Krupovic, M. \& Bamford, D. H. Virus evolution: how far does the double beta-barrel viral lineage extend? Nat Rev Microbiol 6, 941-948, doi:10.1038/nrmicro2033 (2008).

4. San Martin, C. \& van Raaij, M. J. The so far farthest reaches of the double jelly roll capsid protein fold. Virol J 15, 181, doi:10.1186/s12985-018-1097-1 (2018).

5. Liu, L., Cooper, T., Howley, P. M. \& Hayball, J. D. From crescent to mature virion: vaccinia virus assembly and maturation. Viruses 6, 3787-3808, doi:10.3390/v6103787 (2014).

6. Weisberg, A. S. et al. Enigmatic origin of the poxvirus membrane from the endoplasmic reticulum shown by 3D imaging of vaccinia virus assembly mutants. Proc Natl Acad Sci U S A 114, E11001E11009, doi:10.1073/pnas.1716255114 (2017).

7. Chichon, F. J. et al. Membrane remodelling during vaccinia virus morphogenesis. Biol Cell 101, 401414, doi:10.1042/BC20080176 (2009).

8. Chlanda, P., Carbajal, M. A., Cyrklaff, M., Griffiths, G. \& Krijnse-Locker, J. Membrane rupture generates single open membrane sheets during vaccinia virus assembly. Cell Host Microbe 6, 81-90, doi:10.1016/j.chom.2009.05.021 (2009). 
9. Hyun, J. K. et al. Membrane remodeling by the double-barrel scaffolding protein of poxvirus. PLOS Pathog 7, e1002239, doi:10.1371/journal.ppat.1002239 (2011).

10. Bahar, M. W., Graham, S. C., Stuart, D. I. \& Grimes, J. M. Insights into the evolution of a complex virus from the crystal structure of vaccinia virus D13. Structure 19, 1011-1020, doi:10.1016/j.str.2011.03.023 (2011).

11. Garriga, D. et al. Structural basis for the inhibition of poxvirus assembly by the antibiotic rifampicin. Proc Natl Acad Sci U S A 115, 8424-8429, doi:10.1073/pnas.1810398115 (2018).

12. Szajner, P., Weisberg, A. S., Lebowitz, J., Heuser, J. \& Moss, B. External scaffold of spherical immature poxvirus particles is made of protein trimers, forming a honeycomb lattice. J Cell Bio/ 170, 971-981, doi:10.1083/jcb.200504026 (2005).

13. Baldick, C. J., Jr. \& Moss, B. Resistance of vaccinia virus to rifampicin conferred by a single nucleotide substitution near the predicted $\mathrm{NH} 2$ terminus of a gene encoding an $\mathrm{Mr} 62,000$ polypeptide. Virology 156, 138-145, doi:10.1016/0042-6822(87)90444-2 (1987).

14. Charity, J. C., Katz, E. \& Moss, B. Amino acid substitutions at multiple sites within the vaccinia virus D13 scaffold protein confer resistance to rifampicin. Virology 359, 227-232, doi:10.1016/j.virol.2006.09.031 (2007).

15. Mohandas, A. R. \& Dales, S. Involvement of spicules in the formation of vaccinia virus envelopes elucidated by a conditional lethal mutant. Virology 214, 494-502, doi:10.1006/viro.1995.0060 (1995).

16. Sodeik, B. et al. Assembly of vaccinia virus: role of the intermediate compartment between the endoplasmic reticulum and the Golgi stacks. J Cell Bio/ 121, 521-541, doi:10.1083/jcb.121.3.521 (1993).

17. Zhang, Y. \& Moss, B. Immature viral envelope formation is interrupted at the same stage by lac operator-mediated repression of the vaccinia virus D13L gene and by the drug rifampicin. Virology 187, 643-653, doi:10.1016/0042-6822(92)90467-4 (1992).

18. Hyun, J. K. et al. The structure of a putative scaffolding protein of immature poxvirus particles as determined by electron microscopy suggests similarity with capsid proteins of large icosahedral DNA viruses. J Virol 81, 11075-11083, doi:10.1128/JVI.00594-07 (2007).

19. Fang, Q. et al. Near-atomic structure of a giant virus. Nat Commun 10, 388, doi:10.1038/s41467-01908319-6 (2019).

20. Wang, N. et al. Architecture of African swine fever virus and implications for viral assembly. Science 366, 640-644, doi:10.1126/science.aaz1439 (2019).

21. Liu, S. et al. Cryo-EM Structure of the African Swine Fever Virus. Cell Host Microbe 26, 836-843 e833, doi:10.1016/j.chom.2019.11.004 (2019).

22. Xian, Y. \& Xiao, C. Current capsid assembly models of icosahedral nucleocytoviricota viruses. Adv Virus Res 108, 275-313, doi:10.1016/bs.aivir.2020.09.006 (2020).

23. Xian, Y., Karki, C. B., Silva, S. M., Li, L. \& Xiao, C. The Roles of Electrostatic Interactions in Capsid Assembly Mechanisms of Giant Viruses. Int J Mol Sci 20, doi:10.3390/ijms20081876 (2019). 
24. Abrescia, N. G. et al. Insights into assembly from structural analysis of bacteriophage PRD1. Nature 432, 68-74, doi:10.1038/nature03056 (2004).

25. Andres, G., Charro, D., Matamoros, T., Dillard, R. S. \& Abrescia, N. G. A. The cryo-EM structure of African swine fever virus unravels a unique architecture comprising two icosahedral protein capsids and two lipoprotein membranes. J Biol Chem 295, 1-12, doi:10.1074/jbc.AC119.011196 (2020).

26. Reddy, H. K., Carroni, M., Hajdu, J. \& Svenda, M. Electron cryo-microscopy of bacteriophage PR772 reveals the elusive vertex complex and the capsid architecture. Elife 8, doi:10.7554/eLife.48496 (2019).

27. Twarock, R. \& Luque, A. Structural puzzles in virology solved with an overarching icosahedral design principle. Nat Commun 10, 4414, doi:10.1038/s41467-019-12367-3 (2019).

28. Wolf, M., Garcea, R. L., Grigorieff, N. \& Harrison, S. C. Subunit interactions in bovine papillomavirus. Proc Natl Acad Sci U S A 107, 6298-6303, doi:10.1073/pnas.0914604107 (2010).

29. Schur, F. K. et al. An atomic model of HIV-1 capsid-SP1 reveals structures regulating assembly and maturation. Science 353, 506-508, doi:10.1126/science.aaf9620 (2016).

30. Scheres, S. H. RELION: implementation of a Bayesian approach to cryo-EM structure determination. $J$ Struct Biol 180, 519-530, doi:10.1016/j.jsb.2012.09.006 (2012).

31. Zivanov, J. et al. New tools for automated high-resolution cryo-EM structure determination in RELION3. Elife 7, doi:10.7554/eLife.42166 (2018).

32. Zivanov, J., Nakane, T. \& Scheres, S. H. W. Estimation of high-order aberrations and anisotropic magnification from cryo-EM data sets in RELION-3.1. IUCrJ 7, 253-267, doi:10.1107/S2052252520000081 (2020).

33. He, S. \& Scheres, S. H. W. Helical reconstruction in RELION. J Struct Bio/ 198, 163-176, doi:10.1016/j.jsb.2017.02.003 (2017).

34. Zheng, S. Q. et al. MotionCor2: anisotropic correction of beam-induced motion for improved cryoelectron microscopy. Nat Methods 14, 331-332, doi:10.1038/nmeth.4193 (2017).

35. Rohou, A. \& Grigorieff, N. CTFFIND4: Fast and accurate defocus estimation from electron micrographs. J Struct Biol 192, 216-221, doi:10.1016/j.jsb.2015.08.008 (2015).

36. Pettersen, E. F. et al. UCSF Chimera-a visualization system for exploratory research and analysis. $J$ Comput Chem 25, 1605-1612, doi:10.1002/jcc.20084 (2004).

37. Goddard, T. D. et al. UCSF ChimeraX: Meeting modern challenges in visualization and analysis. Protein Sci 27, 14-25, doi:10.1002/pro.3235 (2018).

38. Tan, Y. Z. et al. Addressing preferred specimen orientation in single-particle cryo-EM through tilting. Nat Methods 14, 793-796, doi:10.1038/nmeth.4347 (2017).

39. Wolf, M., DeRosier, D. J. \& Grigorieff, N. Ewald sphere correction for single-particle electron microscopy. Ultramicroscopy 106, 376-382, doi:10.1016/j.ultramic.2005.11.001 (2006).

40. Grant, T., Rohou, A. \& Grigorieff, N. cisTEM, user-friendly software for single-particle image processing. Elife 7, doi:10.7554/eLife.35383 (2018). 
41. Xiong, Q., Morphew, M. K., Schwartz, C. L., Hoenger, A. H. \& Mastronarde, D. N. CTF determination and correction for low dose tomographic tilt series. J Struct Biol 168, 378-387, doi:10.1016/j.jsb.2009.08.016 (2009).

42. Mastronarde, D. N. \& Held, S. R. Automated tilt series alignment and tomographic reconstruction in IMOD. J Struct Bio/ 197, 102-113, doi:10.1016/j.jsb.2016.07.011 (2017).

43. Terwilliger, T. C., Ludtke, S. J., Read, R. J., Adams, P. D. \& Afonine, P. V. Improvement of cryo-EM maps by density modification. Nat Methods 17, 923-927, doi:10.1038/s41592-020-0914-9 (2020).

44. Afonine, P. V. et al. Real-space refinement in PHENIX for cryo-EM and crystallography. Acta Crystallogr D Struct Biol 74, 531-544, doi:10.1107/S2059798318006551 (2018).

45. Emsley, P., Lohkamp, B., Scott, W. G. \& Cowtan, K. Features and development of Coot. Acta Crystallogr D Biol Crystallogr 66, 486-501, doi:10.1107/S0907444910007493 (2010).

46. Afonine, P. V. et al. New tools for the analysis and validation of cryo-EM maps and atomic models. Acta Crystallogr D Struct Biol 74, 814-840, doi:10.1107/S2059798318009324 (2018).

\section{Figures}


a
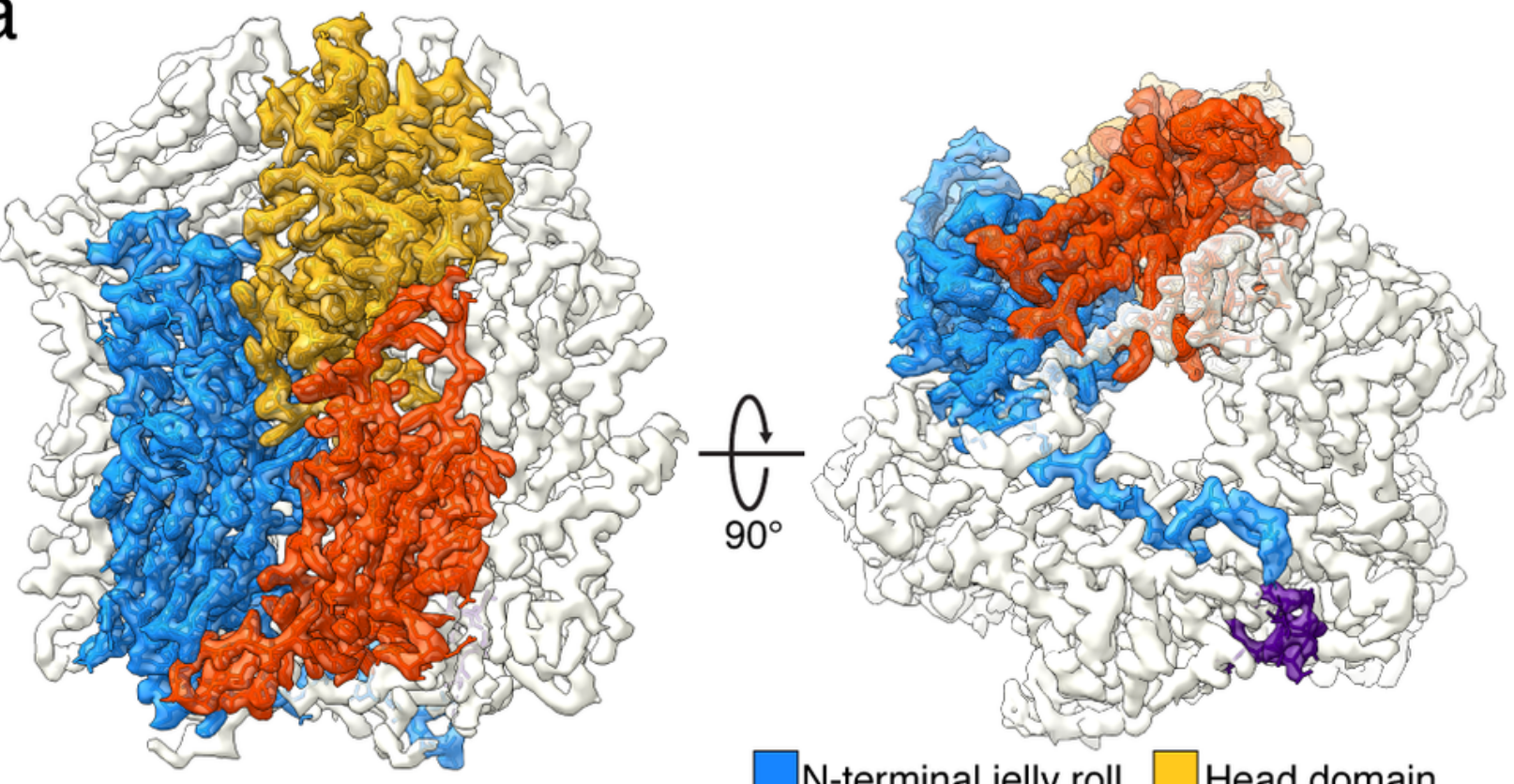

$\square$ N-terminal jelly roll $\square$ Head domain

b

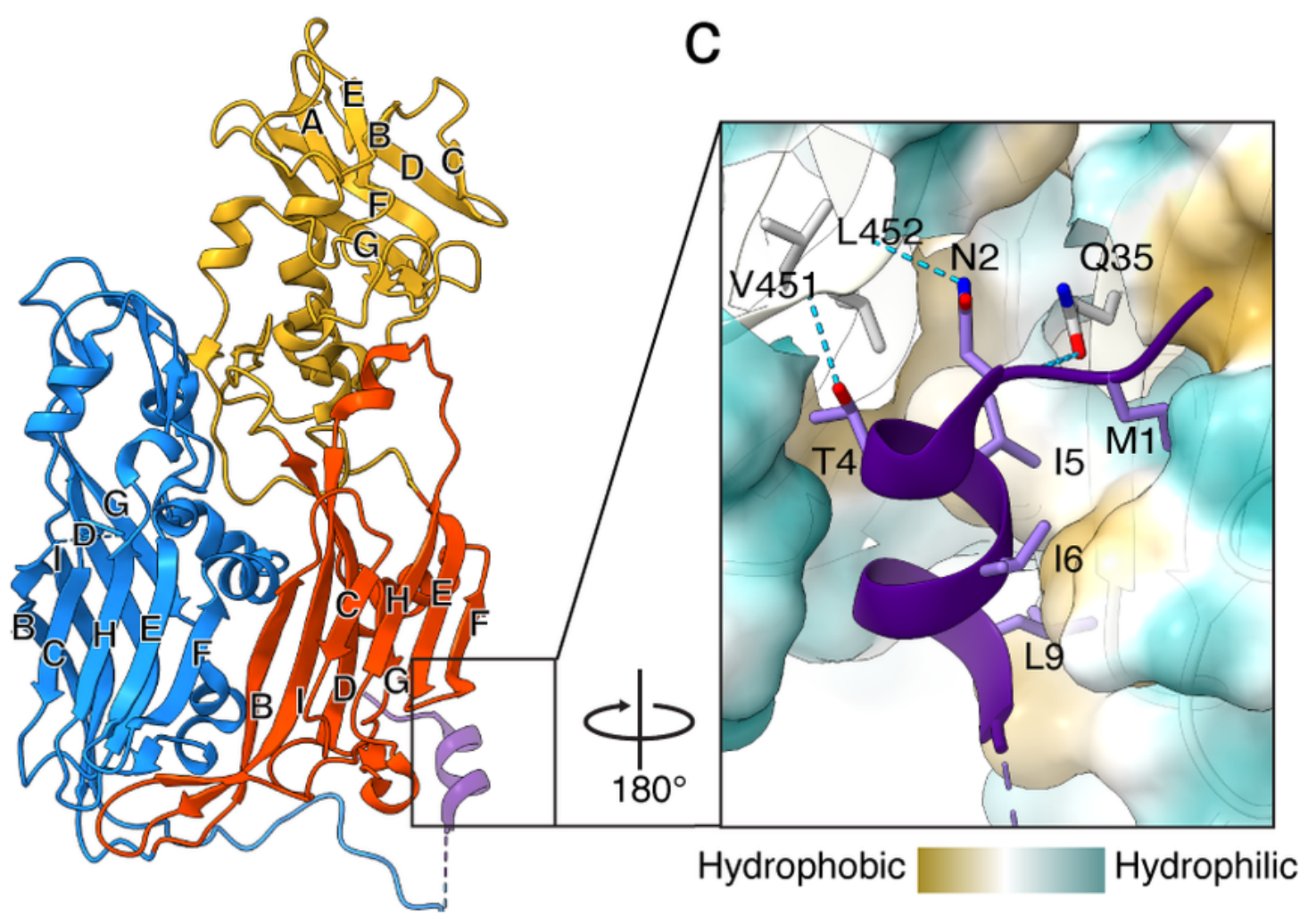

Figure 1

Cryo-EM structures of the VACV D13 trimer a, Iso-electron potential surface of the cryo-EM reconstruction of the D13 trimer contoured at 3.0 0 above average. Map resolution is $2.3 \AA$. The fitted atomic model of one monomer is shown in stick representation within the transparent iso-surface. b, Schematic architecture of the D13 monomer depicted in ribbon representation, composed of two jellyroll domains and one head domain. $\beta$-strands in each domain are labeled in alphabetical order, based on ascending 
amino acid sequence. c, A close-up view of the N-terminal tail a-helix model stabilized in the hydrophobic pocket of a neighboring monomer and hydrogen bonds (dashed cyan line). The Conolly surface of that monomer is colored according to its molecular lipophilicity potential.

a
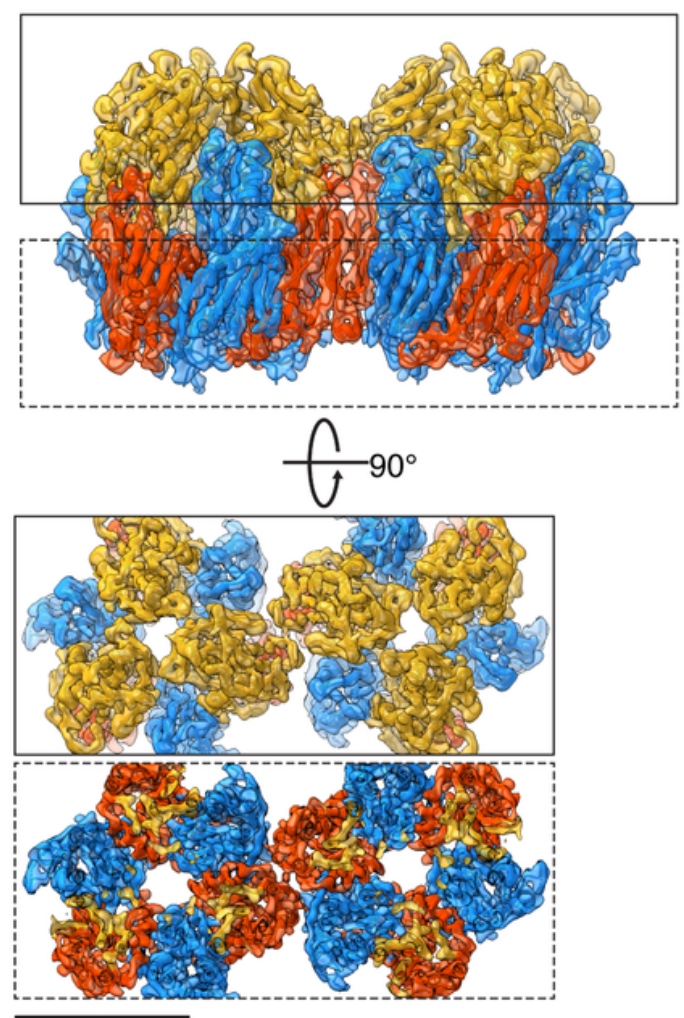

d

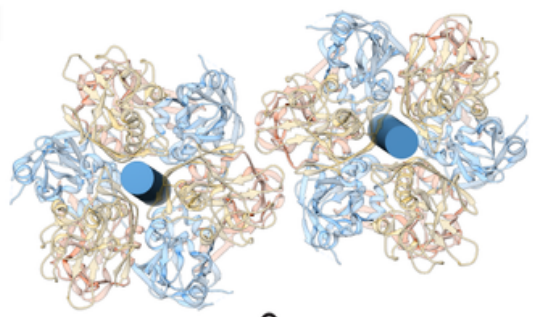

$\frac{1}{y} 90^{\circ}$

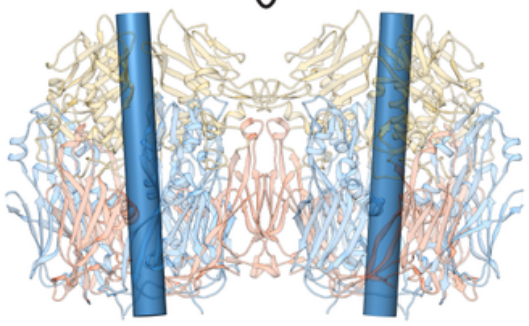

b

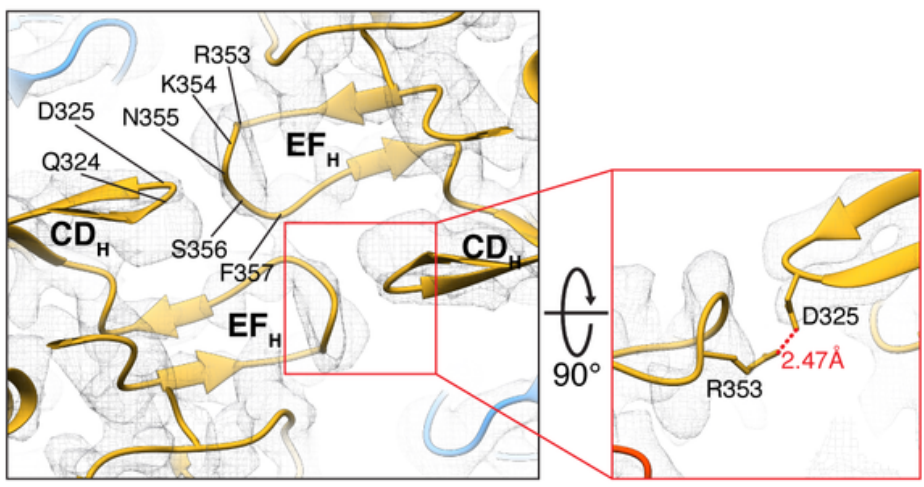

C

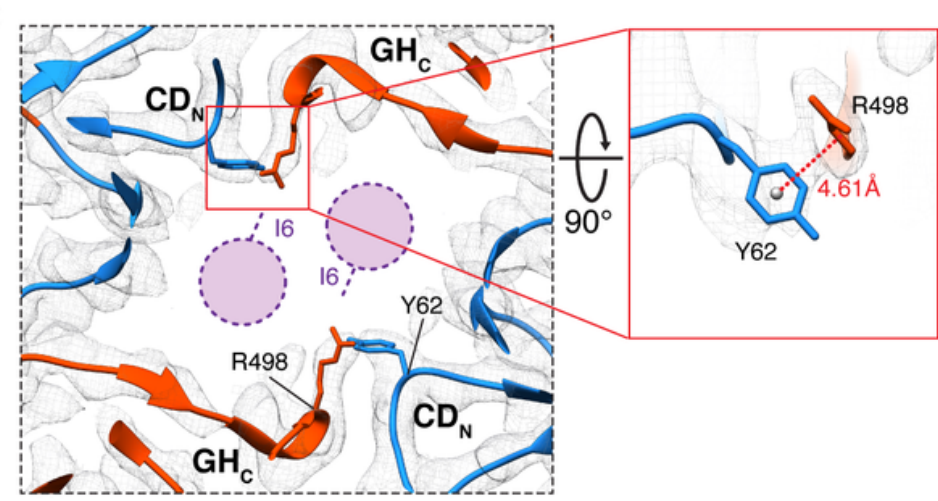

e
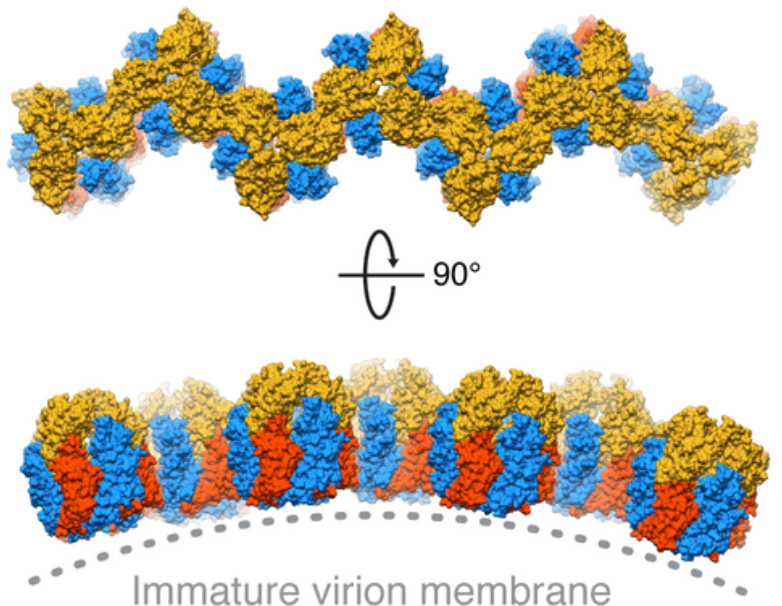

Immature virion membrane
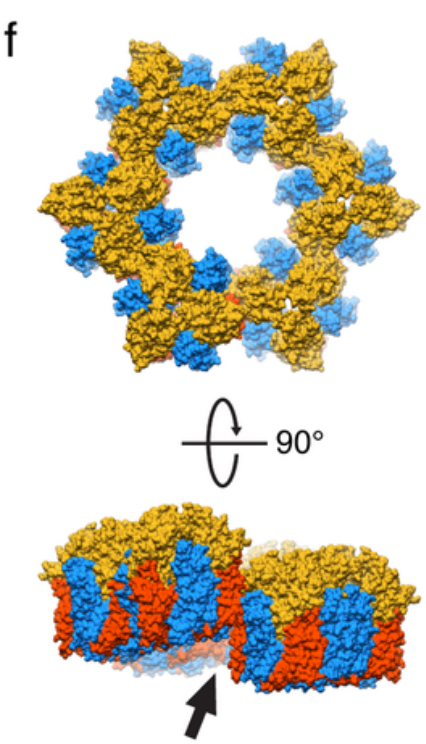

Figure 2

Cryo-EM structures of the VACV D13 trimer doublet a, Iso-electron potential surface (contoured at 3.0 $\mathrm{\sigma}$ above average) of D13 trimers doublet with docked molecular model. Inter-trimer contacts are revealed in slabs (boxes with straight and dashed lines) extracted at different clipping planes. b, Head-to-head contact between the trimers in the doublet model superposed on the cryo-EM map. A potential salt bridge 
interaction between R353 and D325 is shown in a magnified view in a red box. c, Base-to-base contact between trimers in the doublet model superposed on the cryo-EM map. Positions of the missing Nterminal tail a-helix in the doublet structure are depicted as purple circles, with its isoleucine (16) rotamer indicated by a protruding dashed line. Cation- $\pi$ interaction between Y62 and R498 is shown in the red box. $d$, Relative position of neighboring trimers in the doublet as shown by cylinders indicating the 3 -fold symmetry axis of each trimer. The torsion angle between the axes is $8.0^{\circ}$. e, f, Linear and planar expansions of D13 doublet interface. Side view of the linear expansion exhibits convex curvature with the trimer base facing the center of the arch. Planar expansion results in concentric ring of six trimers but the ring does not close (arrow) due to geometrical clash. Scale bars, $5 \mathrm{~nm}$. 

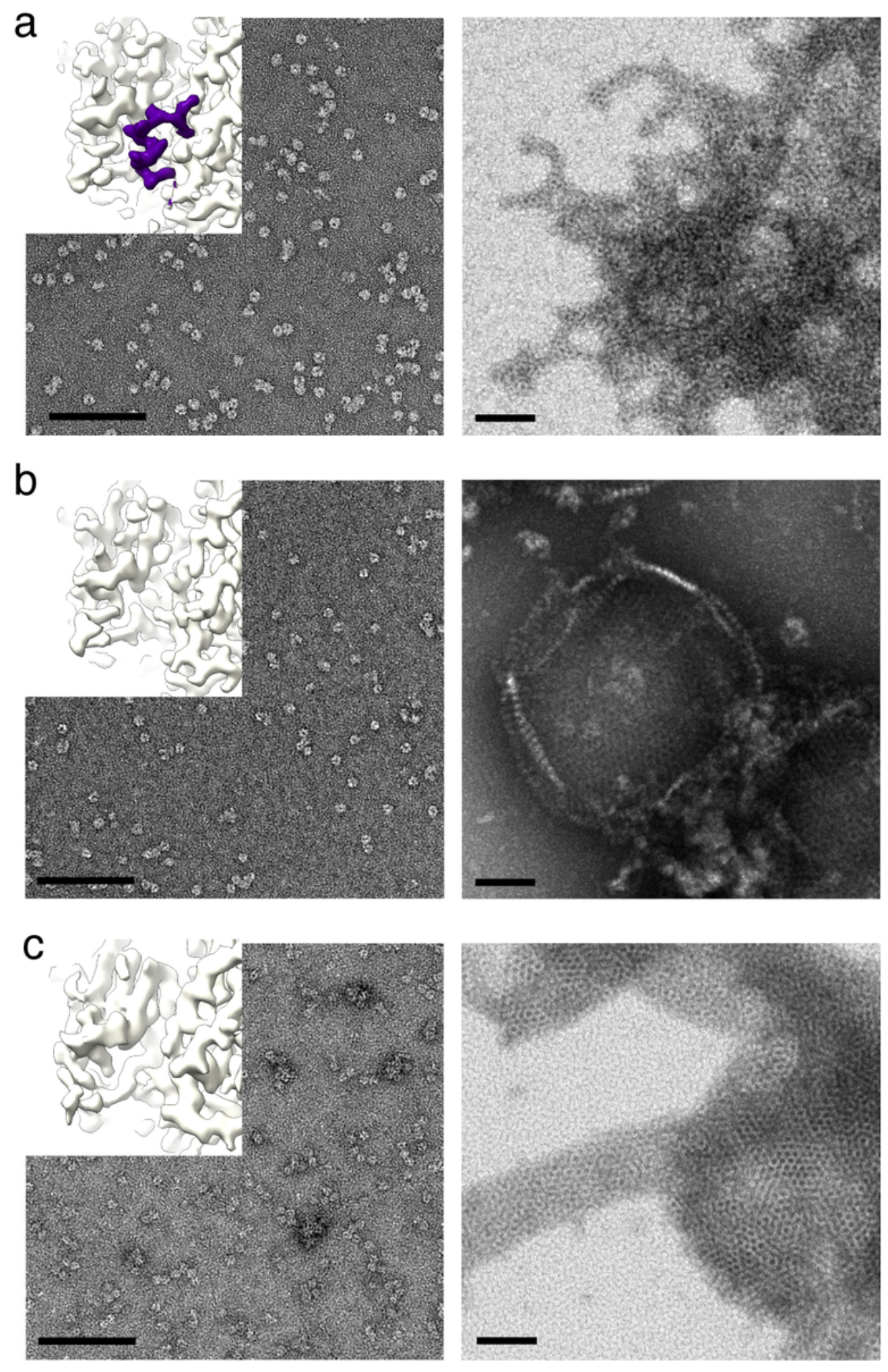

\section{Figure 3}

Assembly of D13 requires displacement of the $\mathrm{N}$-terminal tail a-helix All images are transmission electron micrographs of negatively stained protein before (left column) and after (right column) low-salt, buffermediated assembly of (a) wtD13, (b) His6-tagged D13 and (c) D1318-548. Insets show details of the respective contoured cryo-EM reconstructions. Their structures reveal that the wild-type trimer has an intact $\mathrm{N}$-terminal tail helix (colored purple), whereas the corresponding density is missing in His6-tagged 
protein and in the truncation mutant. Proteins exist as homogenous trimers in $600-\mathrm{mM} \mathrm{NaCl}$ storage buffer. Reduction of the salt concentration to $150 \mathrm{mM}$ leads to aggregation (wtD13) or assembly into spherical (His6-tagged D13) or tubular assemblies (D1318-548). Iso-electron potential surfaces are contoured at $3.0 \sigma$ above the average of cryo-EM reconstructions at resolution $2.3 \AA, 2.6 \AA$ and $4.1 \AA(a, b$, c, respectively). Scale bars, $100 \mathrm{~nm}$.
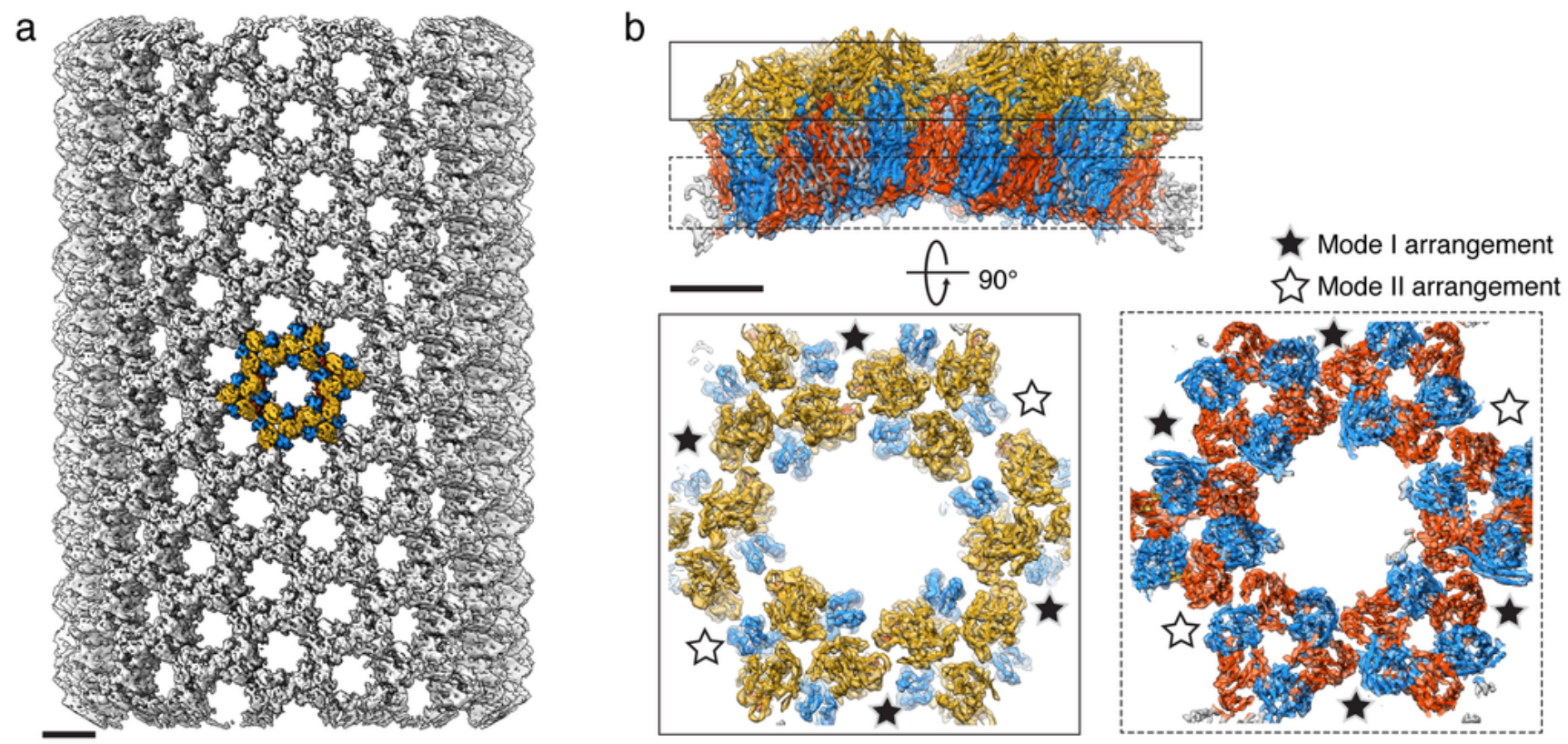

C
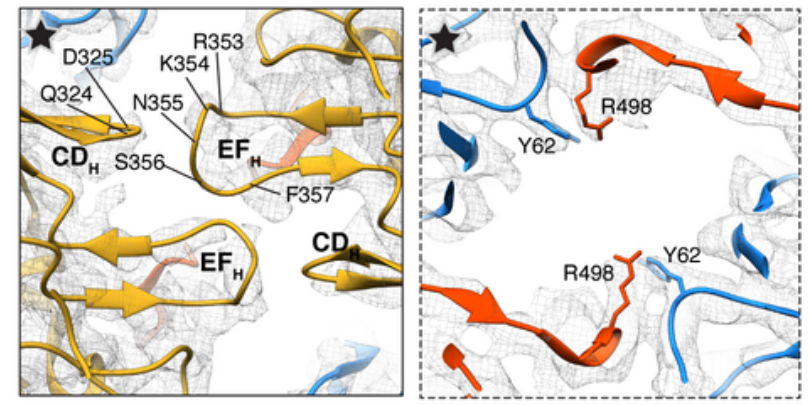

d
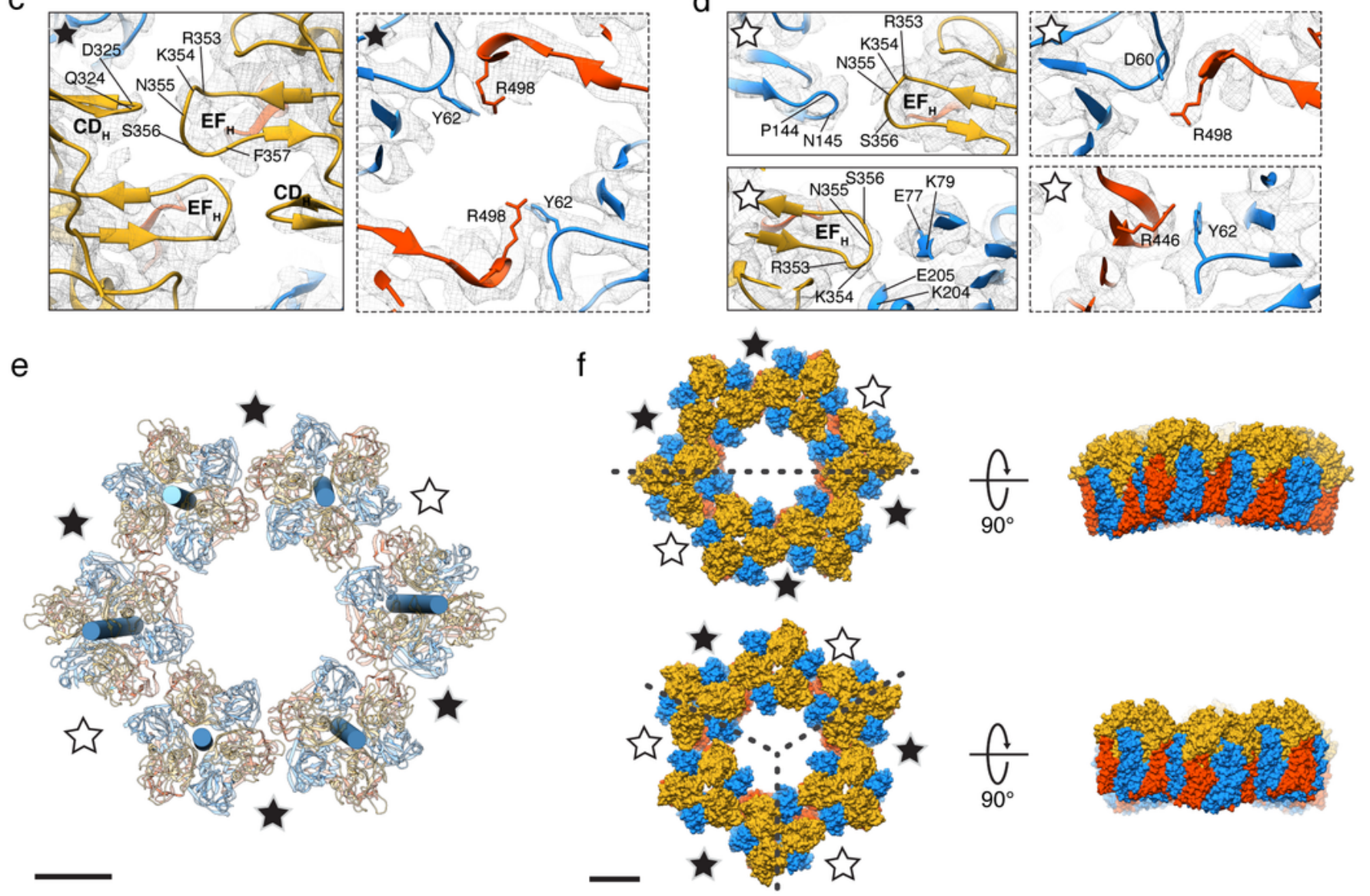

Figure 4 
Structure of a tubular assembly of D13 trimers a, 3D reconstruction of the tubular D13 assembly at $7.3 \AA$ resolution. The map was contoured at $5.0 \sigma$ above average. A hexameric ring of trimers is colored, based on the scheme in Fig. 1. b, 3D reconstruction of a signal-subtracted D13 trimer sextet at $3.9 \AA$ resolution (iso-surface contoured at 5.0 ) with a superposed atomic model. Inter-trimer contacts near the two-fold symmetry axis are revealed within slabs through the reconstruction extracted at different clipping planes (boxes with bold and dashed lines). c, d, Cartoon representation of a molecular model depicting loops and amino acid residues involved in inter-trimer interfaces. Mode I arrangement features two-fold symmetry, whereas contact points in the mode II arrangement are asymmetric. e, Relative position of neighboring trimers in the sextet as shown by cylinders indicating the 3-fold symmetry axis of each respective trimer. $f$, Curvature of $D 13$ trimer sextet found in tubular assembly (upper panel) and manually modeled concentric ring of six trimers with alternating mode I and II arrangement (lower panel). The curvature is unidirectional in tubular assembly along the direction depicted by dashed line whereas alternating modes of arrangements allow for full closure of the ring while generating the curvature around 3 -fold symmetry axis. Scale bars, $10 \mathrm{~nm}$ in a and $5 \mathrm{~nm}$ in b, e, f, respectively. 
a

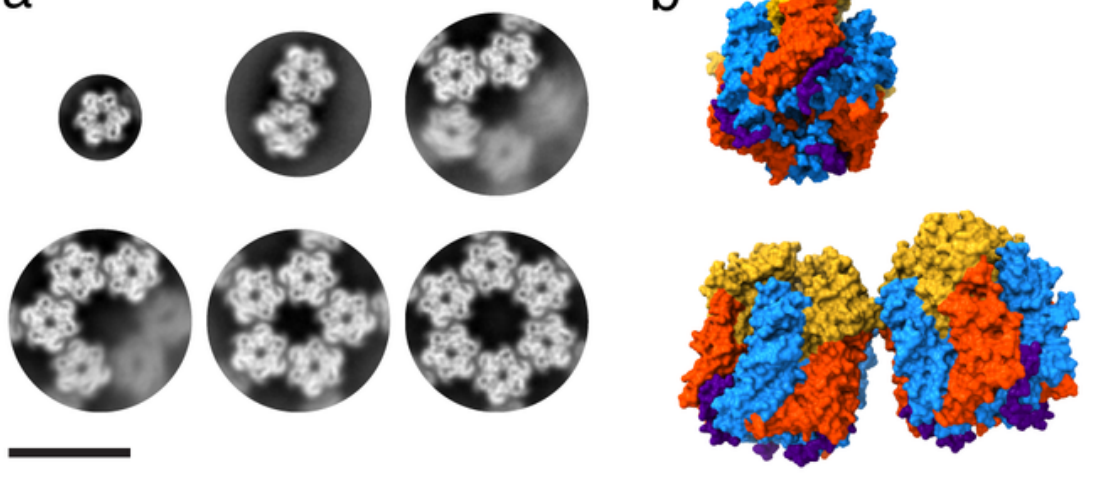

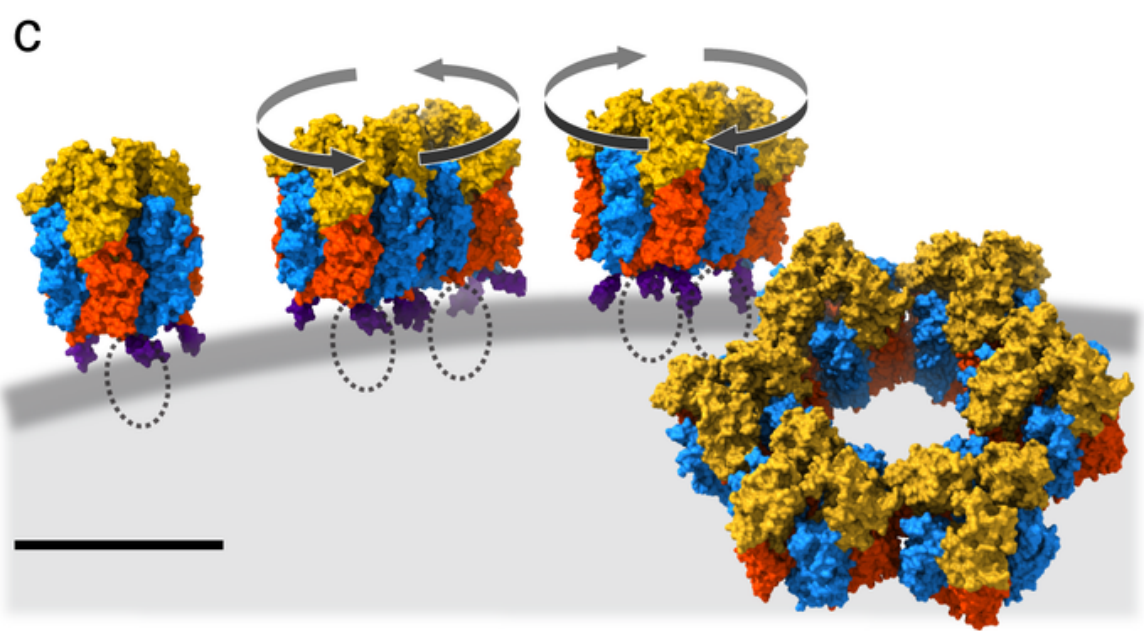

d

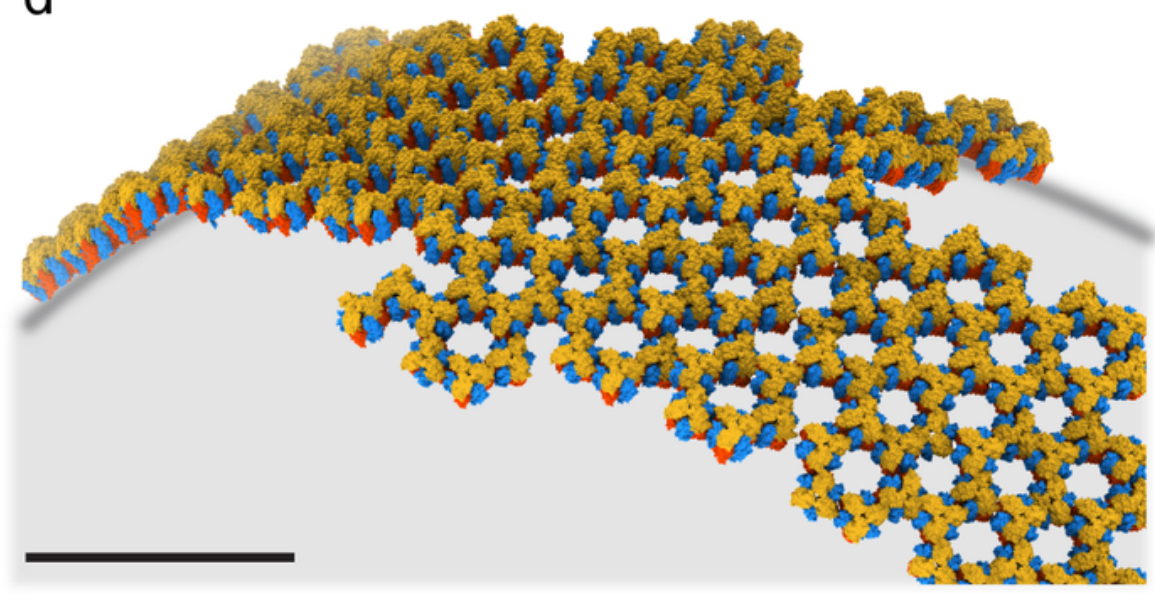

\section{Figure 5}

Capsid-like assembly hypothesis of the immature VACV scaffold a, Gallery of D13 oligomers that are basic building blocks of the honeycomb-shaped lattice. Class averages were generated from electron micrographs of honeycomb-like patches in Supplementary Fig. 1. b, c, Schematic illustration of D13 assembly initiation. D13 in cytoplasm exists as individual trimers, or as randomly associated multimers that are linked only by head-to-head inter-trimer interfaces (b). Once D13 is recruited onto the surface of a 
viral membrane (depicted by a curved grey line) through an interaction with A17 (depicted as dashed ellipses), the N-terminal helix (purple) is dislocated away from D13 and exposes the base-to-base intertrimer interface (c). D13 trimers can subsequently dimerize with two distinct inter-trimer torsions (clockwise and counterclockwise arrows), predetermining the curvature. Continued multimerization of D13 on the viral membrane leads to formation of closed sextets (Fig. 4f, lower panel). d, Expansion of the honeycomb-like lattice into a spherical scaffold. Superposition of trimers on the copies of the sextet model leads to a smoothly curved surface lattice. The curvature is not sufficiently continuous to form a completely spherical morphology, suggesting that additional oligomers such as pentameric building blocks or different combinations of intertrimer arrangements are required to form the authentic immature VACV scaffold. Scale bars, $10 \mathrm{~nm}$ in a, c and $50 \mathrm{~nm}$ in d, respectively.

\section{Supplementary Files}

This is a list of supplementary files associated with this preprint. Click to download.

- NCOMMS2140416TSupplementaryinformation.docx

- SupplementayVideo1.mp4

- NCOMMS2140416Treportingsummary.pdf

- ValidationReports.pdf 